\title{
TRAC, a collaborative computer tool for tracer-test interpretation
}

\author{
A. Gutierrez ${ }^{1, a}$, T. Klinka ${ }^{1}$, D. Thiéry ${ }^{1}$, E. Buscarlet ${ }^{1}$, S. Binet ${ }^{2}$, N. Jozja ${ }^{3}$, C. Défarge ${ }^{2,3}$, \\ B. Leclerc ${ }^{4}$, C. Fécamp ${ }^{2}$, Y. Ahumada ${ }^{5}$ and J. Elsass ${ }^{6}$ \\ ${ }^{1}$ BRGM, Service Eau, BP. 36009, 45060 Orléans Cedex 2, France \\ ${ }^{2}$ Université d'Orléans, Université François Rabelais - Tours, CNRS/INSU, Institut des Sciences de la Terre \\ d'Orléans - UMR 6113, Polytech'Orléans, 45072 Orléans Cedex 2, France \\ ${ }^{3}$ Université d'Orléans, CETRAHE, (CEllule R\&D d'Expertise et de TRAnsfert en TRAçages Appliqués à \\ l'Hydrogéologie et à l'Environnement), 45072 Orléans Cedex 2, France \\ ${ }^{4}$ EDREE Parc des Aulnaies, 84 rue du Beuvron, 45160 Olivet, France \\ ${ }^{5}$ Institut EGID, Bordeaux 3 - 1, allée F. Daguin, 33607 Pessac Cedex, France \\ ${ }^{6}$ Software development. circular@operamail.com
}

\begin{abstract}
Artificial tracer tests are widely used by consulting engineers for demonstrating water circulation, proving the existence of leakage, or estimating groundwater velocity. However, the interpretation of such tests is often very basic, with the result that decision makers and professionals commonly face unreliable results through hasty and empirical interpretation. There is thus an increasing need for a reliable interpretation tool, compatible with the latest operating systems and available in several languages. BRGM, the French Geological Survey, has developed a project together with hydrogeologists from various other organizations to build software assembling several analytical solutions in order to comply with various field contexts. This computer program, called TRAC, is very light and simple, allowing the user to add his own analytical solution if the formula is not yet included. It aims at collaborative improvement by sharing the tool and the solutions. TRAC can be used for interpreting data recovered from a tracer test as well as for simulating the transport of a tracer in the saturated zone (for the time being). Calibration of a site operation is based on considering the hydrodynamic and hydrodispersive features of groundwater flow as well as the amount, nature and injection mode of the artificial tracer. The software is available in French, English and Spanish, and the latest version can be downloaded from the web site http://trac.brgm.fr.
\end{abstract}

\section{INTRODUCTION}

Contaminant migration in aquifers threatens groundwater quality and the exploitability of water-supply wells. Tracer tests are often used for assessing the vulnerability of groundwater bodies, as well as the velocity of contaminant flow and its pathways. Tracer test interpretation also provides hydrodispersive parameters that are used as input for flow and transport models.

In France, tracer tests are widely used for defining protection zones around water-supply wells; their interpretation generally relies on abacus $[1,2]$, or uses the simple CATTI [3] program running under MS-DOS.

The increasing demand for such expertise and the growing interest in health and environmental problems requires new and improved methods.

Since the end of the $19^{\text {th }}$ Century, many experiments and studies have been carried out to understand and simulate transport, adsorption, desorption, degradation and reaction of solutes in variably saturated underground media. Analytical or numerical models were developed for determining the solute concentration versus time and distance from the original contamination point. However, the few dedicated tools for tracer-test interpretation are not evolving. Interpretation of a tracer test consists in deducing parameters from the evolution of concentration over time, at an observation point located at a certain distance from the injection point. These parameters, generally designed as hydrodispersive parameters, include transit

\footnotetext{
a e-mail: a.gutierrez@brgm.fr
}

time, kinematic porosity, dispersion, retardation factor, first-order degradation coefficient, etc., which are used for a simple characterization of the behaviour of a perfectly water-miscible solute in movement towards or within groundwater bodies. Tracer-test analysis requires drawing a concentration versus time graph, called the breakthrough curve. Its interpretation can follow two different ways:

- From an initial asumption of flow and transport parameters, the computation of a theoretical breakthrough curve aims at getting a good fit with the observed one. This is the direct problem;

- Hydrodispersive parameters are directly computed from the observed concentrations. This is the inverse problem.

The computation codes for interpreting a breakthrough curve discussed here use an analytical solution derived from the general convection-dispersion equation to evaluate solute transport in porous media. This equation can be written as follows: considering $C(x, t)$ as the volumetric mass concentration of the contaminant and $q_{w}$ the flux velocity vector, if no adsorption reactions occur between the contaminant and the surrounding porous medium, then the transport is determined by convection and diffusion. The mass conservation law implies that

$$
\begin{aligned}
f(x, t)= & \phi \frac{\partial C(x, t)}{\partial t}+\nabla \cdot(D \nabla C(x, t)) \\
& -q w \cdot \nabla C(x, t)
\end{aligned}
$$

This is an Open Access article distributed under the terms of the Creative Commons Attribution License 2.0, which permits unrestricted use, distribution, and reproduction in any medium, provided the original work is properly cited. 
Table 1. Comparison of several codes used in tracer test interpretation.

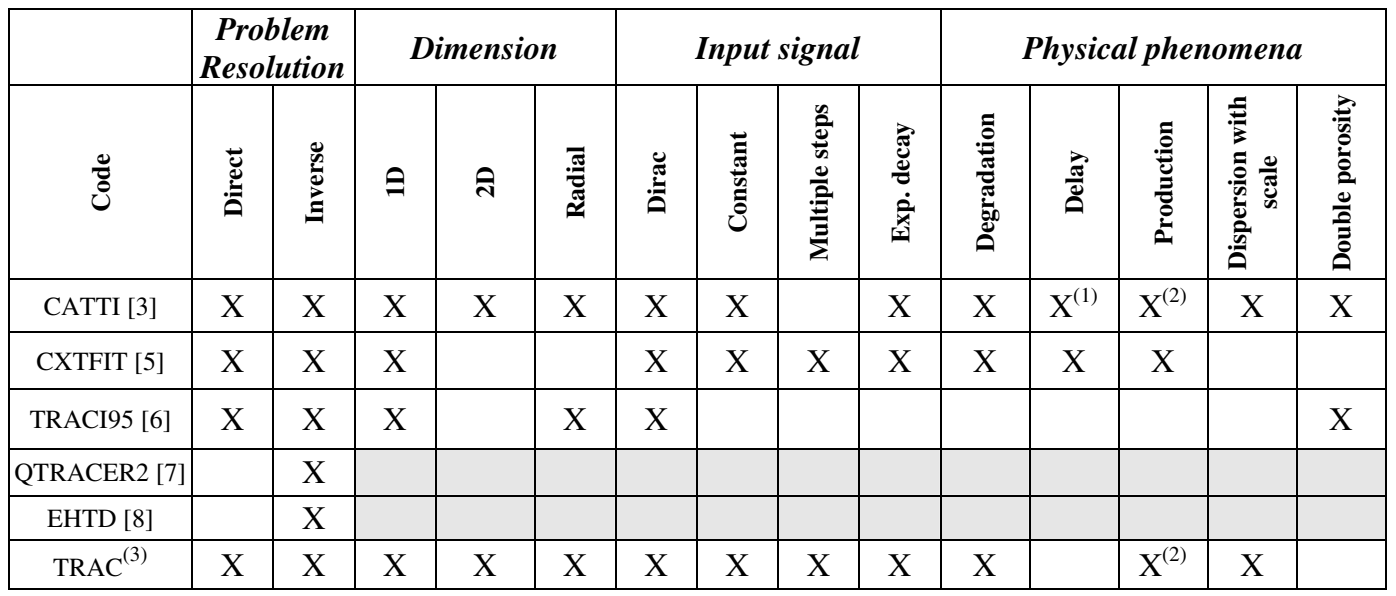

(1) CATTI considers a constant delay with the Dirac injection type.

(2) Production corresponds here to an initial concentration in the aquifer (initial noise).

(3) TRAC version 1.3.8 presented in this paper.

where, $f(x, t)$ is the external contamination-source rate of injection, $\phi$ is the kinematic porosity, and D stands for the diffusion tensor. The second and third terms of the equation correspond to Fick's first law, which assumes that the contaminant flux is proportional to the local concentration gradient. The second term is known as the diffusive flux and the third one the convective (or advective) flux.

Considering adsorption, the mass balance equation becomes

$$
\begin{aligned}
f(x, t)= & \frac{\partial(\phi C(x, t)+\rho S(x, t))}{\partial t}+\nabla \cdot(D \nabla C(x, t)) \\
& -q_{w} \cdot \nabla C(x, t)
\end{aligned}
$$

where $\rho$ corresponds to the bulk density of soil and $S$ is the adsorbed mass concentration. $S$ is linked to concentration through a linear adsorption isotherm of the form

$$
S(x, t)=k_{d} C(x, t)
$$

where $\mathrm{k}_{\mathrm{d}}$ is an empirical distribution coefficient.

Taking equation (3) into account, equation (2) can be written as:

$$
\begin{aligned}
f(x, t)= & R \frac{\partial C(x, t)}{\partial t}+\nabla \cdot(D \nabla C(x, t)) \\
& -q_{w} \cdot \nabla C(x, t)
\end{aligned}
$$

where $R$ is the retardation factor (dimensionless):

$$
R=\phi+\rho k_{d} .
$$

Several tools derived from this general equation are compared hereafter. They usually share the following hypothesis:

- The porous medium is homogeneous,

- Flow is uniform or radial,

- Tracer injection may be instantaneous injection (Dirac) or continuous (constant concentration step). Some of the tools consider successive injection steps of various concentrations (multi-step) or exponential decay.
Tools that allow considering degradation or sorption/desorption phenomena of a tracer in a porous medium are less numerous. CATTI [3] proposes a solution to account for the scale dependency of longitudinal dispersivity typically encountered in applied field problems [4].

Table 1 summarizes the main characteristics of software used for tracer-test interpretation. Most run under DOS mode and are incompatible with the latest operating systems. The TRAC software, presented in this paper, has been added to the table below.

\section{SOFTWARE DESCRIPTION}

TRAC is software developed with the objective of filling the lack of computer tools for interpreting tracer tests. Its aim is to remain both simple, in order to correspond to basic engineering needs, and advanced with an option that enables an evolved mode where solutions that are more complex are available. It operates based on analytical solutions that can be selected depending on the configuration of the tracer test. Up to now, the following scenarios are available:

- 1D, 2D, radial converging/diverging flow;

- Instantaneous (Dirac), continuous or step-injection;

- Possibility of taking into account a delay factor and a degradation constant (exponential form);

- Space (i.e. time) variable dispersivity in 1D to consider the scale effect;

- Variable direction of flow in order to respect the uncertainty of groundwater flow direction;

- Possibility to do a multi-step injection for all solutions with continuous injection (use of the superposition principle).

Tracer-testing methods continue to evolve and new interpretation methods are being developed. TRAC gives experienced users the possibility to introduce their own analytical solution. TRAC can thus be continuously updated and enhanced by contributions of the user community. 


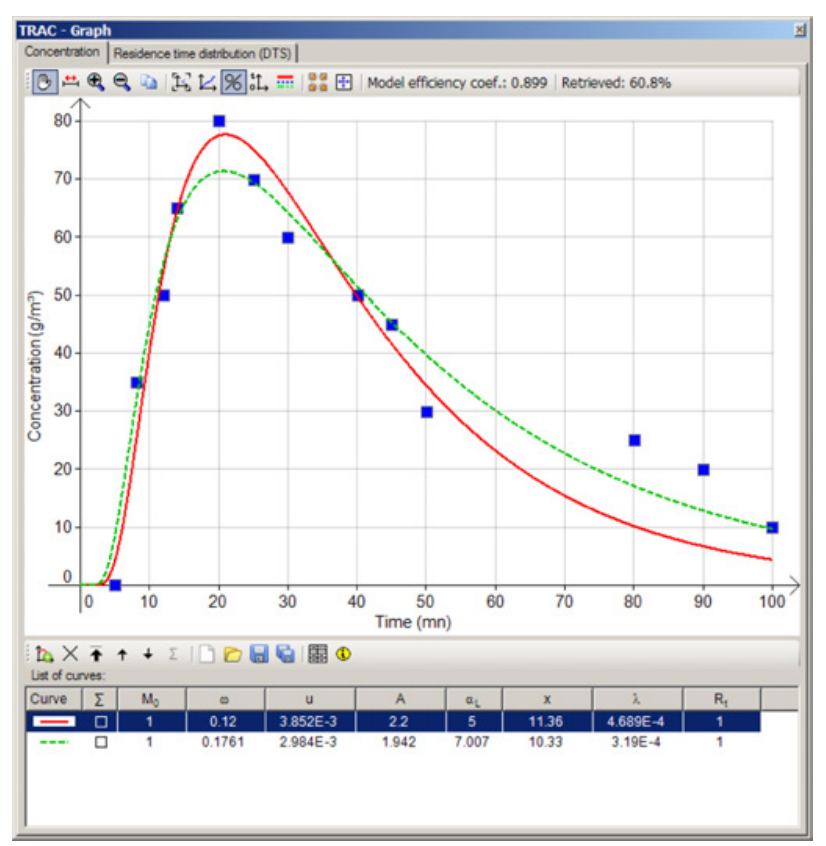

Figure 1. Screenshot of the TRAC graphic window.

TRAC's start-up offers two modes: "simulate and design" or "interpret" field-tracer tests or soil-column experiments.

The "simulation" mode, used for preparing a tracer test, helps estimating the optimal mass or concentration to be injected and the sampling frequency at the restitution point.

The "interpretation" mode consists in determining the hydrodispersive parameters of the medium by calibration. This is done by selecting one of the available analytical solutions and, from a set of parameters fixed by the user and a range of possible values, the software search for an optimized best fit (Fig. 1). Every parameter can be set up for optimization or not. TRAC lets the user define whether he wants to optimize a specific parameter or not, and in which range he accepts this parameter to vary.

Once the interpretation is done, the printing options allow a synthetic representation of the tracer-test interpretation, including a summary of the parameters or a plot of the cumulative restitution curve.

\section{ANALYTICAL SOLUTIONS}

The present list of analytical solutions (TRAC v1.4.4) amounts to 14 in the advanced mode, but only 12 have been validated up to now. The other ones are in the validation process, which consists in comparing the results of a set of parameters with several other tools against TRAC. Other tools can be Excel ${ }^{\circledR}$ or Fortran ${ }^{\circledR}$ programs of the analytical solution, and mathematical modelling using the MARTHE finite-volume code [17]. The list of symbols and notations used in the following formulas is given below (Table 2).
Table 2. Symbols and notations used in the formulas.

$\alpha_{T} \quad(\mathrm{~m})[\mathrm{L}]=$ Transversal dispersivity.

$C_{O} \quad\left(\mathrm{~kg} / \mathrm{m}^{3}\right)[\mathrm{M} / \mathrm{L} 3]=$ Injection concentration of tracer.

C $\quad\left(\mathrm{kg} / \mathrm{m}^{3}\right)[\mathrm{M} / \mathrm{L} 3]=$ Concentration of tracer at point $(\mathrm{x}, \mathrm{y}, \mathrm{z})$ and time $(\mathrm{t})$.

$D_{L} \quad\left(\mathrm{~m}^{2} / \mathrm{s}\right)[\mathrm{L} 2 / \mathrm{T}]=$ Longitudinal dispersion $\left(D L=\alpha L^{*} u\right)$

$D_{T} \quad\left(\mathrm{~m}^{2} / \mathrm{s}\right)[\mathrm{L} 2 / \mathrm{T}]=$ Transversal dispersion $\left(D T=\alpha T^{*} u\right)$.

$e \quad(\mathrm{~m})[\mathrm{L}]=$ Aquifer thickness.

$M_{0}(\mathrm{~kg})[\mathrm{M}]=$ Mass of injected tracer.

$\omega \quad(-)[-]=$ Effective porosity $(0<\omega<1)$.

$\mathrm{Pe} \quad(-)[-]=$ Péclet number $(\mathrm{Pe}=\mathrm{x} / \alpha)$.

$P_{\mathrm{L}} \quad$ (s) $[\mathrm{T}]=$ Time period for approaching the asymptotic value of $\alpha \mathrm{L}$.

$\begin{array}{ll}P_{\mathrm{T}} & (\mathrm{s})[\mathrm{T}]=\text { Time period for approaching }\end{array}$ the asymptotic value of $\alpha \mathrm{T}$.

$q \quad(\mathrm{~m} 3 / \mathrm{s})[\mathrm{L} 3 / \mathrm{T}]=$ Injection rate of tracer.

$q_{m} \quad(\mathrm{~kg} / \mathrm{s})[\mathrm{M} / \mathrm{T}]=$ Massic injection rate of tracer.

$Q_{p} \quad(\mathrm{~m} 3 / \mathrm{s})[\mathrm{L} 3 / \mathrm{T}]=$ Pumping rate.

$\begin{array}{ll}r & (\mathrm{~m})[\mathrm{L}]=\text { Radial distance injection }- \text { restitution. }\end{array}$

$R_{\mathrm{t}} \quad(-)[-]=$ Delay factor.

$\theta \quad\left({ }^{\circ}\right)[-]=$ Angle between the direction of flow and the $\mathrm{x}$-axis.

$t \quad(\mathrm{~s})[\mathrm{T}]=$ Time.

$t_{1 / 2}(\mathrm{~s})[\mathrm{T}]=$ Half-life $(\mathrm{t} 1 / 2=\ln (2) / \lambda)$.

$u \quad(\mathrm{~m} / \mathrm{s})[\mathrm{L} / \mathrm{T}]=$ Pore velocity.

$v \quad(\mathrm{~m} / \mathrm{s})[\mathrm{L} / \mathrm{T}]=$ Darcy's velocity $\left(\mathrm{v}=\mathrm{u}^{*} \omega\right)$.

$x \quad(\mathrm{~m})[\mathrm{L}]=$ Distance between measurement and injection points regarding the $\mathrm{x}$-axis.

y $\quad(\mathrm{m})[\mathrm{L}]=$ Distance between measurement and injection points regarding the y-axis.

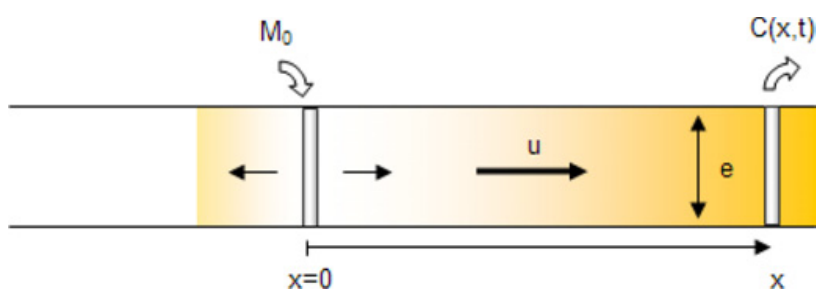

Figure 2. Brief mass injection in an infinite medium.

\subsection{Uniform 1D flow}

\section{Brief injection}

\section{Brief mass injection in an infinite medium (1)}

This solution corresponds to a brief and punctual injection of mass $M_{0}$ per cross-sectional unit of aquifer transverse to the flow $(A=e * 1)$, of thickness e and unit width (Fig. 2). The tracer mass is injected at time $t=0$ at point $x=0$. The tracer moves in terms of the pore velocity in the medium.

If the decay constant $\lambda$ is defined, the concentration of the transported element is subjected to an exponential decrease of type $C_{0} \cdot e^{-\lambda t}$ during transport. This solution is modified from $[9,10]$.

$$
C(x, t)=\frac{M_{0}}{A \omega \sqrt{4 \pi D_{L} t}} \exp \left[-\frac{(x-u t)^{2}}{4 D_{L} t}\right] \exp (-\lambda t) .
$$




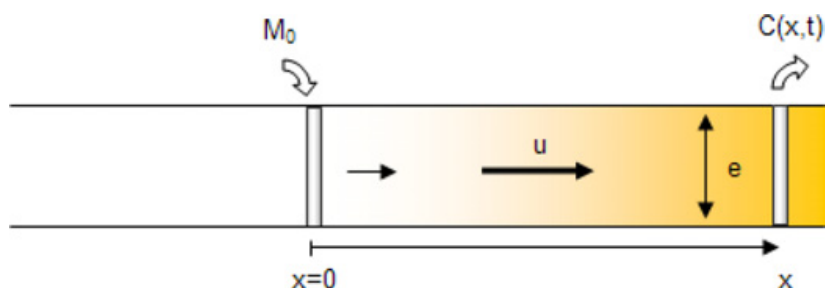

Figure 3. Brief mass injection in a semi-infinite medium.

\section{Brief mass injection in a semi-infinite medium (2)}

This solution corresponds to a brief injection of mass $M_{0}$ per cross-sectional unit of aquifer transverse to the flow ( $A=e * 1$ ), of thickness e and unit width (Fig. 3). The tracer mass is injected at time $t=0$ at point $x=0$. The tracer moves in terms of the pore velocity in the medium. If the decay constant $\lambda$ is not defined, the transported element is not subjected to degradation. Otherwise, the degradation is analogue to solution (1). This solution is modified from [19].

$$
\begin{aligned}
C(x, t)= & \frac{M_{0}}{A \omega}\left[\frac{2}{\sqrt{4 \pi D_{L} t}} \exp \left(-\frac{(x-u t)^{2}}{4 D_{L} t}\right)\right. \\
& \left.-\frac{u}{2 D_{L}} \exp \left(\frac{u x}{D_{L}}\right) \operatorname{erfc}\left(\frac{(x+u t)}{\sqrt{4 D_{L} t}}\right)\right] \exp (-\lambda t) .
\end{aligned}
$$

\section{Continuous injection}

Continuous injection of a constant concentration in an infinite medium (3)

This solution corresponds to continuous injection of a constant concentration at $x=0$, at time $t=0$ in an infinite medium (Fig. 4).

If the decay constant $\lambda$ is not defined the transported element is not subjected to degradation. Otherwise, the degradation is analogue to solution (1).

If the concentration remains constant during injection, the injected mass over the time interval $\Delta \mathrm{t}$ is $\mathrm{M}=$ $\mathrm{C}_{0}$. .u.u.A. $\Delta \mathrm{t}$. The scheme is similar as in solution (1), except that injection is constant. This solution is modified from [16].

$$
\begin{aligned}
C(x, t)= & \frac{C_{0}}{2}\left[\operatorname{erfc}\left(\frac{x-u t}{\sqrt{4 D_{L} t}}\right)-\exp \left(\frac{u x}{D_{L}}\right)\right. \\
& \left.\times \operatorname{erfc}\left(\frac{x+u t}{\sqrt{4 D_{L} t}}\right)\right] \exp (-\lambda t) \\
C_{0}= & \frac{q_{m}}{A \omega u} .
\end{aligned}
$$

Continuous injection of a constant concentration in a semiinfinite medium (4)

This solution corresponds to continuous injection of a constant concentration in a semi-infinite medium (Fig. 5).

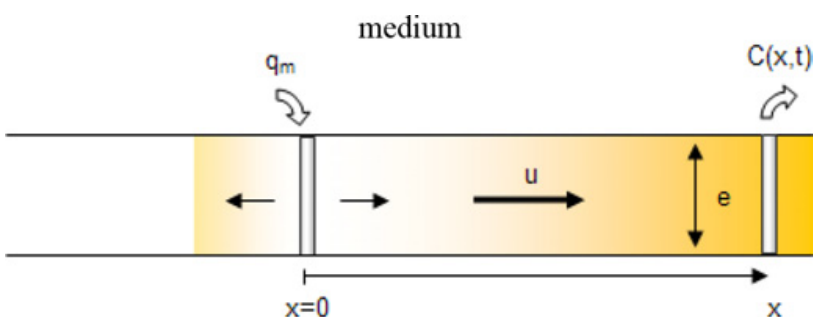

Figure 4. Injection of a constant concentration in an infinite medium.

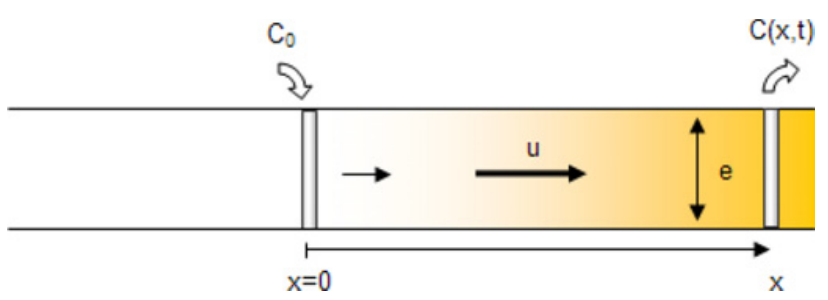

Figure 5. Injection of a constant concentration in a semi-infinite medium.

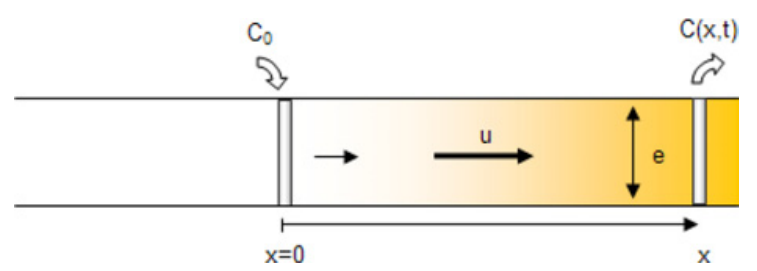

Figure 6. Continuous injection of a constant concentration in a semi-infinite medium.

If the decay constant $\lambda$ is not defined, the transported element is not subjected to degradation. Otherwise, the degradation is analogue to solution (1). This solution is modified from $[16,20]$.

$$
\begin{aligned}
C(x, t)= & \frac{C_{0}}{2}\left[\operatorname{erfc}\left(\frac{(x-u t)}{\sqrt{4 D_{L} t}}\right)+\exp \left(\frac{u x}{D_{L}}\right)\right. \\
& \left.\times \operatorname{erfc}\left(\frac{x+u t}{\sqrt{4 D_{L} t}}\right)\right] \exp (-\lambda t) .
\end{aligned}
$$

Continuous injection of a constant concentration in a semi-infinite medium with exponential decay in the aquifer (5)

This solution corresponds to continuous injection of a constant concentration in a semi-infinite medium (Fig. 6). The concentration of the transported element exponentially decreases in groundwater following the relation $C_{s}=$ $C_{0} e^{-\lambda t}$, and the source concentration (injection point) is not subjected to degradation and remains constant. This formulation may be used for simulating, as an example, a bacterial degradation occurring only in the groundwater and not in the injection well. This solution 


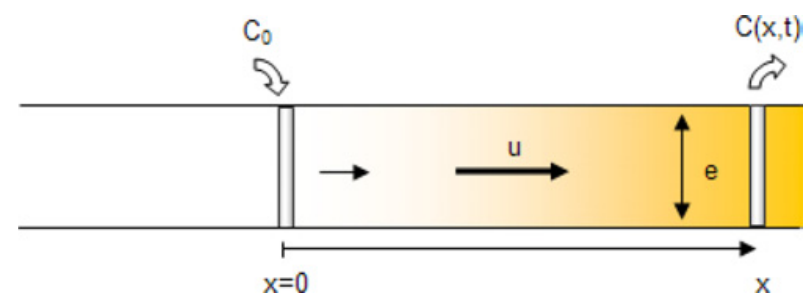

Figure 7. Continuous injection of a constant concentration in a semi-infinite medium.

is ([11]):

$$
\begin{aligned}
& C(x, t)=\frac{C_{0}}{2}\left[\exp \left[\frac{(u-\Gamma) x}{2 D_{L}}\right] \operatorname{erfc}\left(\frac{x-\Gamma t}{\sqrt{4 D_{L} t}}\right)\right. \\
& \left.+\exp \left[\frac{(u+\Gamma) x}{2 D_{L}}\right] \operatorname{erfc}\left(\frac{x+\Gamma t}{\sqrt{4 D_{L} t}}\right)\right] \\
& \Gamma=u \sqrt{1+\frac{4 \lambda D_{L}}{u^{2}}} .
\end{aligned}
$$

The decay term of the element during transport becomes null if the decay constant $\lambda=0$, so $\Gamma=\mathrm{u}$.

Continuous injection of a constant concentration in a semi-infinite medium with exponential decay of the source term (6)

This solution corresponds to continuous injection of a constant concentration in a semi-infinite medium, with or without degradation of the transported element (Fig. 7). In this case, exponential degradation of the element occurs both in the groundwater and in the source, as opposed to the previous solution where decay occurs solely in groundwater. This solution is modified from $[12,13]$.

$$
\begin{aligned}
& C(x, t)=\frac{C_{0}}{2}\left[\operatorname{erfc}\left(\frac{x-u t}{\sqrt{4 D_{L} t}}\right)-\exp \left(\frac{u x}{D_{L}}\right) \operatorname{erfc}\left(\frac{x+u t}{\sqrt{4 D_{L} t}}\right)\right. \\
& \left.\times\left[1+\frac{u(x+u t)}{D_{L}}\right]+\frac{2 u t}{\sqrt{\pi D_{L} t}} \exp \left[-\frac{(x-u t)^{2}}{4 D_{L} t}\right]\right] \exp (-\lambda t) .
\end{aligned}
$$

\subsection{Uniform 2D flow}

\section{Brief injection}

\section{Brief injection of a mass in a semi-infinite medium (7)}

This solution corresponds to a brief injection of mass $M_{O}$ per aquifer thickness unit, with or without degradation of the transported element (exponential decay) (Fig. 8). The injection occurs at point $(x, y)=(0,0)$, the concentration is computed at point $(x, y)$. The flow direction is assumed parallel to the $\mathrm{x}$-axis; if it is not the case the next solution may be more accurate, as it allows specifying

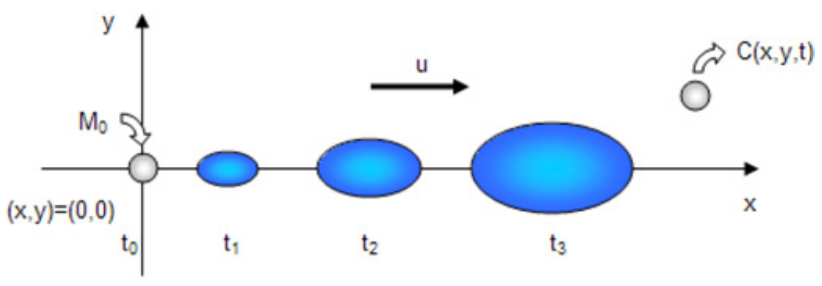

Figure 8. Brief injection of mass in a $2 \mathrm{D}$ infinite medium.

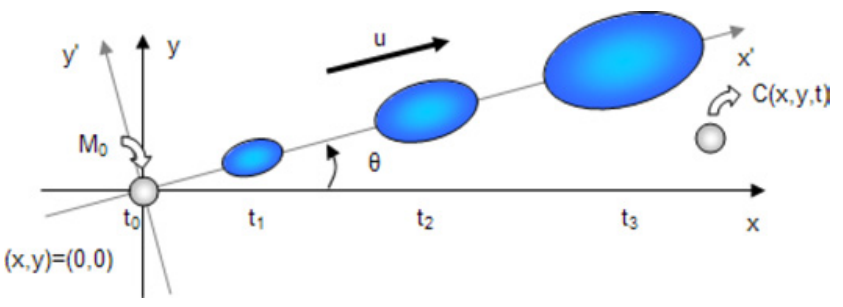

Figure 9. Brief injection of mass in a 2D infinite medium, with a specified direction of the flow.

an angle for the flow direction. This solution is modified from [14]:

$$
\begin{aligned}
C(x, y, t)= & \frac{M_{0}}{4 \pi e \omega t \sqrt{D_{L} D_{T}}} \exp \left[-\frac{(x-u t)^{2}}{4 D_{L} t}-\frac{y^{2}}{4 D_{T} t}\right] \\
& \times \exp (-\lambda t) .
\end{aligned}
$$

Brief injection of mass in an infinite medium with flow direction (8)

This solution is a modified version of the previous one, corresponding to a brief injection of mass $\mathrm{M}_{0}$ per aquifer thickness unit, including the direction of flow $\theta$ diverging from the $\mathrm{x}$-axis (Fig. 9). This solution is modified from [14]:

$$
\begin{aligned}
C(x, y, t)= & \frac{M_{0}}{4 \pi e \omega t \sqrt{D_{L} D_{T}}} \exp \left[-\frac{\left(x^{\prime}-u t\right)^{2}}{4 D_{L} t}-\frac{y^{\prime 2}}{4 D_{T} t}\right] \\
& \times \exp (-\lambda t) \\
\text { with } \quad x^{\prime}= & x \cos \theta+y \sin \theta \\
y^{\prime}= & x \sin \theta+y \cos \theta .
\end{aligned}
$$

Brief injection of mass in a semi-infinite medium with variable dispersivity (9)

This solution corresponds to a brief injection of mass $M_{O}$ per aquifer thickness unit, subjected to a constant flux directed parallel to the $\mathrm{x}$-axis (injection axis restitution point). This analytical solution incorporates the observed growth of dispersivity values with flow time or flow distance. The scale effect is considered by using time-dependent dispersivity values. This solution with varying dispersivity values requires two additional input parameters $\left(P_{L}\right.$ and $\left.P_{T}\right)$. 


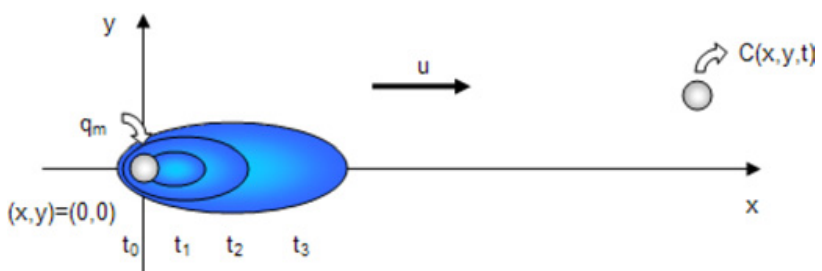

Figure 10. Constant injection of mass flux in a $2 \mathrm{D}$ infinite medium.

The longitudinal and transversal dispersivity increase with distance of transport and inflect to become asymptotic to a maximum value. $P_{L}$ and $P_{T}$ are the two fitting parameters representing the time needed to reach the asymptotic value of the longitudinal and transversal dispersivity $\left(\alpha_{L}\right.$ and $\left.\alpha_{T}\right)$.

The scheme of this solution is analogue to that of Figure 8. This solution is ([15]):

$$
\begin{aligned}
C(x, y, t)= & \frac{M_{0}}{2 \pi e \omega \sigma_{L}(t) \sigma_{T}(t)} \exp \left[-\frac{(x-u t)^{2}}{2 \sigma_{L}^{2}(t)}-\frac{y^{2}}{2 \sigma_{T}^{2}(t)}\right] \\
& \times \exp (-\lambda t)
\end{aligned}
$$

with

$$
\begin{aligned}
& \sigma_{L}^{2}(t)=2 \alpha_{L} u t\left[1+\frac{P_{L}}{t}\left(\exp \left(-\frac{t}{P_{L}}\right)\right)-1\right] \\
& \sigma_{T}^{2}(t)=2 \alpha_{T} u t\left[1+\frac{P_{T}}{t}\left(\exp \left(-\frac{t}{P_{T}}\right)\right)-1\right] .
\end{aligned}
$$

\section{Continuous injection}

Continuous injection of mass flux in an infinite medium (10)

This solution corresponds to a continuous injection of concentration at a defined injection rate (mass flux: $q_{m}=$ q.C0, mass per unit of time and per unit of aquifer thickness) in an infinite medium (Fig. 10). This solution is ([3]).

$$
\begin{aligned}
& C(x, y, t)=\frac{q C_{0}}{4 \pi e \omega u \alpha_{L} \alpha_{T}} \\
& \times \int_{0}^{t} \frac{1}{t} \exp \left(-\frac{(x-u t)^{2}}{4 \alpha_{L} u t}-\frac{y^{2}}{4 \alpha_{T} u t}\right) \exp (-\lambda t) d t .
\end{aligned}
$$

Continuous injection of mass flux in an infinite medium with variable dispersion (11)

This solution is a modified version of the previous one that includes dispersivity values increasing over flow time. This solution is ([3]):

$$
\begin{aligned}
C(x, y, t)= & \frac{q C_{0}}{2 \pi e \omega} \\
& \times \int_{0}^{t} \frac{\exp \left(\frac{(x-u t)^{2}}{2 \sigma_{L}^{2}(t)}-\frac{y^{2}}{2 \sigma_{T}^{2}(t)}\right) \exp (-\lambda t)}{\sigma_{L}(t) \sigma_{L}(t)} d t
\end{aligned}
$$
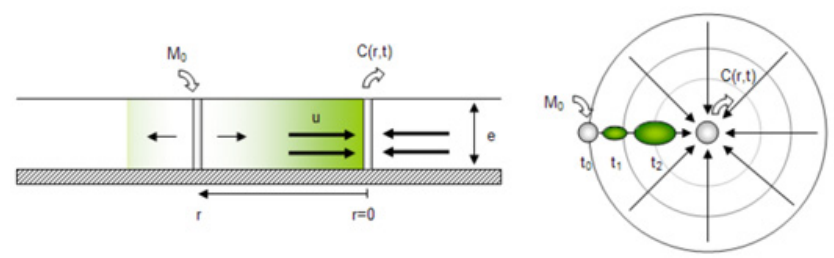

Figure 11. Brief injection in a radial convergent flow.

with

$$
\begin{aligned}
& \sigma_{L}^{2}(t)=2 \alpha_{L} u t\left[1+\frac{P_{L}}{t}\left(\exp \left(-\frac{t}{P_{L}}\right)\right)-1\right] \\
& \sigma_{T}^{2}(t)=2 \alpha_{T} u t\left[1+\frac{P_{T}}{t}\left(\exp \left(-\frac{t}{P_{T}}\right)\right)-1\right] .
\end{aligned}
$$

The integral upside can be computed numerically, but the results may be inaccurate for high $P_{L}$ and $P_{T}$ values (over 10 days).

\subsection{Radial converging flow}

\section{Brief injection}

Brief injection of mass in a radial convergent flow induced by a continuous pumping well (12)

This solution corresponds to a tracer test in a radial convergent flow induced by a continuous pumping well $(Q p)$ (Fig. 11). Though until now no exact analytical solution exists, an approximate solution is given by ([18]):

$$
C(x, t)=\frac{M_{0} r}{2 Q_{p} t^{3 / 2} \sqrt{\pi \alpha_{L} u}} \exp \left(-\frac{(r-u t)^{2}}{4 D_{L} t}\right) \exp (-\lambda t) .
$$

The tracer is injected in a lateral observation well located at a distance $r$ from the pumping well. This solution is:

The Péclet number is the relation between the advective and diffusive transfer:

$$
P e=\frac{r}{\alpha_{L}} .
$$

Note that the approximate solution should be used for Péclet numbers larger than three, i.e. for cases where the distance from the injection point to the pumping well is at least three times as large as the longitudinal dispersivity $\left(r>3 \alpha_{L}\right)$.

To address this problem, a new and more accurate semi-analytical solution is being developed and will be added to the next release of TRAC. This solution will allow simulating a tracer in converging (pumping well) or diverging (injection well) radial flow even for smaller Péclet numbers.

\section{VALIDATION AND EXAMPLE}

TRAC has been compared to other ways of computation, either using a spreadsheet program such as EXCEL or 


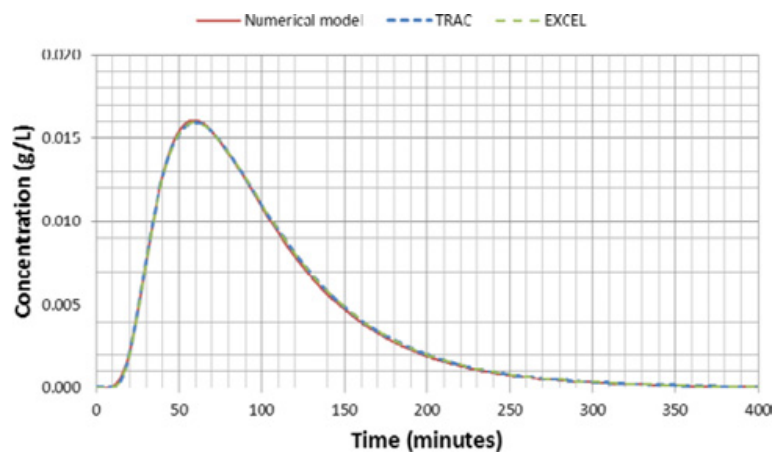

Figure 12. Comparison between TRAC, EXCEL and a numerical model, MARTHE on a 1D simulation (Eq. (7)).

a specific development of the analytical solution using FORTRAN language. The objective of these comparisons was to ensure a proper implementation of the formulas in the code. No differences at all were noticed. This validates the tool, but does not validate the formula, which is however supposed to be valid within the limits of its application field, as most of the formulas were already tested in the referenced publications from where they come.

In order to validate the formulas as well, numerical simulations were conducted using a finite volume numerical model, MARTHE [21]. The studied domain is discretised in meshes. Grid extension and boundary conditions depends on the type of problem. The validation procedure and results are published in a specific report [22]. We present below (Fig. 12) a breakthrough curve obtained from the simulation of a brief injection (Dirac) of 1 gram of uranine in a semi-infinite medium such as a saturated column which would have the following characteristics:

- Section (area) $=1$ sq.m

- porosity $=10 \%$;

- permeability $=10-5 \mathrm{~m} / \mathrm{s}$;

- hydraulic gradient $=1 \mathrm{~m} / \mathrm{m}$

- longitudinal dispersivity $=0.1 \mathrm{~m}$;

- distance injection-restitution $=0.5 \mathrm{~m}$.

The model is run in transient mode using a 6 second timestep and a total duration of 10 hours.

The results of this simulation perfectly match the results of analytical solution (2), equation (7) whether it is written within TRAC or with EXCEL.

Another example is given with Figure 13. In this real tracer test performed in an alluvial aquifer, the tracer (uranine) was injected in a piezometer located at $13.9 \mathrm{~m}$ from a pumping well. The pumping discharge at $39.5 \mathrm{~m} 3 / \mathrm{h}$ induces a radial flux to the well. Aquifer thickness is $6.25 \mathrm{~m}$. The tracer was injected in the saturated zone and homogenized along the whole length of the borehole screen before being flushed. The breakthrough curve is interpreted with formula (12) equation (18) for radial converging flow. The results of the tracer experiment are iteratively fitted using the optimization procedure to give the following values:

Longitudinal dispersivity $=0.64 \mathrm{~m}$; Porosity $=0.20$.

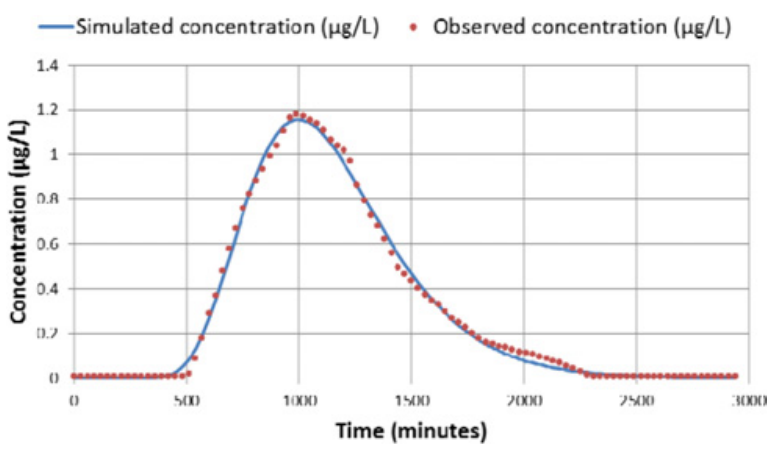

Figure 13. Observed and computed breakthrough curve using Eq. (18) (Radial converging flow).

We verify that the Péclet number $(13.9 / .64=21.7)$ is greater than 3 .

Other examples are given in the user manual [23].

\section{CONCLUSIONS}

The advection-dispersion solute-transport equation quantitatively describes the transport of solutes in groundwater. Analytical solutions that are exact mathematical solutions for this partial differential equation were derived for different combinations (aquifer geometry, boundary, solute source, initial conditions).

TRAC is a simple tool that operates based on validated analytical solutions for simulating solute transport in one-, two- and radial-dimensional systems with uniform flow. The solutions were compiled from those published in the literature and used in some software. TRAC is user-friendly and its GUI is intuitive. Freely available and downloadable, TRAC may benefit from the use by all interested hydrogeologists, who can continuously improve it by introducing their own analytical solutions.

For more information and access, please see our web site: http://trac.brgm.fr

\section{References}

[1] J.P. Sauty - Mise au point et utilisation d'abaques pour l'interprétation des expériences de traçages dans les nappes d'eau souterraine. Bulletin du BRGM, Section III, n 4-197, pp. 285-291 (1978)

[2] J.P. Sauty - Utilisation des traceurs pour définir les périmètres de protection. Rapport BRGM n ${ }^{\circ} 87$ SGN-287-EAU (1987)

[3] J.P. Sauty, W. Kinzelbach, A. Voss - Computer Aided Tracer Test Interpretation (CATTI), program documentation, International Ground Water Modeling Center, Golden, Colorado, 64 p. (1992)

[4] W.L. Nieman, C.W. Rovey II - A systematic fieldbased testing program of hydraulic conductivity and dispersivity over a range in scale. Hydrogeology Journal 17, 307-320 (2009)

[5] N. Toride, F.J. Leij, M.Th. van Genuchten - The CXTFIT code for estimating transport parameters from laboratory or field tracer experiments. Version 
2.0, Research Report $n^{\circ}$ 137, US Salinity Laboratory (1995)

[6] W. Käss - Tracing Technique in Geohydrology, A. A. Balkema, Rotterdam, Brookfield, Vt., 581 p. (1998)

[7] M. Field - The QTRACER2 program for tracerbreakthrough curve analysis for tracer tests in karstic aquifers and other hydrologic systems. EPA/600/R02/001 (2002)

[8] M. Field - Tracer-Test planning using the efficient hydrologic tracer-test design (EHTD) program. EPA/600/R-03/034 (2003)

[9] J. Crank - The mathematics of diffusion. $347 \mathrm{pp}$. London: Oxford University Press (1956)

[10] J. Bear -. Dynamics of fluids in porous media. New York: Elsevier (1972)

[11] M.Th. van Genuchten - Analytical solutions for chemical transport with simultaneous adsorption, reo-order production and first order decay. Journal of Hydrology 49, 213-233 (1981)

[12] N.D. Gershon, A. Nir - Effects of boundary conditions of models on tracer distribution in flow through porous mediums: Water Resources Research 5, 830-839 (1969)

[13] K.H. Coats, B.D. Smith - Dead-end pore volume and dispersion in porous media. Pet. Trans AIME, 231 SPEJ 73, 73-84 (1964)

[14] J.J. Fried -, Groundwater pollution, New York: American Elsevier, 930 p. (1975)

[15] G.T. Csanady - Turbulent Diffusion in the Environment, Dordrecht: Reidel (1973)

[16] J.P. Sauty, Contribution à l'identification des paramètres de dispersion dans les aquifers par l'interprétation des expériences de traçage. Thèse Doct.-Ing. Grenoble (1977)

[17] D. Thiéry, Modélisation 3D du transport de masse avec le logiciel MARTHE Version 5.4. BRGM Report R38149 HYT/DR 95, pp. 171 (1995)

[18] J.P. Sauty, Identification des paramètres du transport hydrodispersif dans les aquifères par interprétation de traçages en écoulement cylindrique convergent ou divergent, Journal of Hydrology 39 (1-2), 69-103 (1978)

[19] M. De Vries in "Tracer Techniques in Sediment Transport. Int. Atomic Energy Agency I.A.E.A., Vienna (1973), 105-123

[20] A. Ogata, R.B. Banks - A solution of the differential equation of longitudinal dispersion in porous media. US Geological Survey, Professional paper 411-A (1961)

[21] D.Thiéry - Modélisation des aquifères complexes Prise en compte de la zone non saturée et de la salinité. Calcul des intervalles de confiance. Revue Hydrogéologie. no. 4, 325-336

[22] T. Klinka, A. Gutierrez. Validation du logiciel TRAC : Aide à l'interprétation de traçages en milieu poreux. BRGM report RP-59425-FR (in French) available on the Trac web site and at http://www.brgm.fr/publication.jsp (2012)

[23] A. Gutierrez, T. Klinka, D. Thiery. Manuel d'utilisation de TRAC : Aide à l'interprétation de traçages en milieux poreux. BRGM report RP60660-FR (in French) available on the Trac web site and at http://www.brgm.fr/publication.jsp (2012) 